\title{
Pyomyositis in Children
}

\author{
Sanjay Verma ${ }^{1}$
}

Published online: 15 February 2016

(C) Springer Science+Business Media New York 2016

\begin{abstract}
Primary pyomyositis in children is prevalent in the tropics and increasingly being recognised from temperate regions. Staphylococcus aureus remains the principle causative organism worldwide, while proportion of communityacquired methicillin-resistant Staphylococcus aureus (CAMRSA) is on rise. Commonly involved groups are around the pelvis and lower limbs. Clinical presentation depends on the stage of disease, most commonly a child presenting with limping with fever. Early diagnosis and management are crucial. The investigation of choice is MRI scan. Appropriate antibiotic treatment should be instituted at the earliest opportunity along with drainage. Majority of patients show excellent and complete recovery with no long-term complications once treatment is started early. A high level of awareness and suspicion of this condition is warranted from all paediatric clinicians.
\end{abstract}

Keywords Pyomyositis · Tropical · Children ·

Staphylococcus aureus $\cdot$ Muscle abscess

This article is part of the Topical Collection on Pediatric Infectious Diseases

Sanjay Verma

sanjay06verma@yahoo.com

1 Department of Pediatrics, Advanced Pediatric Centre, Postgraduate Institute of Medical Education and Research, Chandigarh 160012, India

\section{Introduction}

Pyomyositis in children is not a common manifestation of bacterial infections, but when it occurs, the consequences to the patient may be severe or even fatal. Staphylococcus aureus (S. aureus) is the most likely causative organism, although several other infectious agents, including viruses, fungi, and parasites have also been linked to its causation. This review primarily focuses on its classical presentation, typically named as tropical pyomyositis (TP).

This pathological entity termed 'spontaneous acute myositis' was first described in the mid-nineteenth century by Virchow, but the first description in literature could be traced to a description by Japanese surgeon Scriba in 1885, as an endemic disease in the tropics [1]. Later, another Japanese surgeon, Miyake, extensively reviewed this and added 33 more cases [2]. During the early twentieth century, while British and other European countries expanded colonisation, this disease was recognised with increasing frequency in the native populations and in the soldiers who lived in the tropical areas of Asia and Africa [3]. During this time, it acquired the name by which it is now known widely-tropical pyomyositis. The suitability of this designation was confirmed by another epidemiological study from East Africa, which discovered that the disease was found commonly only in regions with a truly topical climate, i.e. a fairly constant high temperature and high relative humidity $[4 \cdot \bullet]$. In this review, we discuss current epidemiology, clinical manifestation, diagnosis and potential treatment options for tropical pyomyositis in children.

\section{Prevalence}

Tropical pyomyositis has been widely reported from Asia, tropical Africa, Oceania and the Caribbean islands [3, 4••]. 
There are numerous reports of similar cases from Northern India, showing the peak incidence during months of high humidity ranging from July to October [5•, 6-9]. It accounts for 1 to $4 \%$ of all hospital admissions in some tropical countries $[3,5 \cdot]$.

Similar cases are increasingly being reported from temperate regions as well [10-12]. A recent study from Brisbane, Australia, shows increasing prevalence of TP, from 2.04 to 8.73 cases per 10,000 (peak rate 13.5 cases per 10,000 in 2008) $[13 \bullet \cdot]$. Another study from the UK supports the increasing incidence of TP [14•]. There are reports of pyomyositis cases from diverse geographic regions of the USA. In a 10year review, one or two cases of pyomyositis per 4000 paediatric admissions occurred annually in the USA [15]. It was more common in children who live in the southernmost regions of the USA (e.g. Texas) than in children who live in the northern regions (e.g. Iowa) [16].

\section{Epidemiology and Pathogenesis}

Possible mechanism for pyomyositis has been extensively studied under experimental conditions [2]. When healthy rabbits were given boluses of Staphylococcus intravenously, they occasionally developed small abscesses in the liver, kidney or spleen but never in the skeletal muscles. When some injury was caused to specific muscles by mechanical pinching or electric current 24 or $48 \mathrm{~h}$ before the intravenous injection of bacteria, small abscesses developed within few days at injury site in nearly half of them. No abscesses formed in healthy muscles tissues. The role of trauma in causation of pyomyositis was supported further by one study in the British Army [3]. When this disease was observed more commonly in army soldiers, they investigated 32 cases and made several observations. Majority of men recalled having experienced trauma at the affected site; the incidence of abscesses increased as the severity of physical training increased and abscesses were more common on the dominant (right) side of the body [3]. Another analysis of 78 cases in Uganda observed the similar finding of more involvement of the right side of the body [17].

From experimental evidence and clinical observations, two associations with pyomyositis were found: muscle injury and bacteraemia, usually staphylococcal. It was postulated that uninjured skeletal muscle is intrinsically resistant to infection because myoglobin binds to iron avidly, which is necessary for the growth of an organism. But when muscle trauma is present, there is sequestration of elemental iron leading to predisposition for haematogenous invasion by bacteria with subsequent abscess formation [18]. The initial episode of trauma resulted in a staphylococcal skin lesion and, possibly, a bacteraemia that seeded sites of previously bruised muscle. Sources of muscle trauma have ranged from bicycle accidents to strenuous aerobic exercises [19]. These cases also may explain the predilection of the disease to occur in warmer humid climates, concomitant skin infections and muscle trauma, which is more likely to occur in a climate in which children can play or work outside wearing fewer cloths for most of the year. The clustering of cases of TP during humid and rainy seasons (July to October) of the year has been widely reported from Northern India and other tropical countries [5•, 6-9, 20].

The aetiological agent of pyomyositis in more than $90 \%$ of cases is $S$. aureus both in tropical and temperate climates. An increasing percentage is caused by community-acquired methicillin-resistant $S$. aureus (CA-MRSA). There are reports of increasing proportion of CA-MRSA clones worldwide including India, Australia and the USA [13••, 21•]. PantonValentine leukocidin (PVL) is a bacterial exotoxin secreted by Staphylococcus strains that are either methicillin sensitive or methicillin resistant. This toxin is known to cause leukocyte destruction and tissue necrosis in children. There is suggestion in the literature to request PVL status of all $S$. aureus isolates in suspected musculoskeletal sepsis [22•]. Recent study from Texas, USA, showed an increase in the prevalence of CAMRSA in cases of pyomyositis. Of the 24 available S. aureus isolates, 15 (63\%) were CA-MRSA. CA-MRSA, USA300, $p v l$-positive $S$. aureus isolates caused more severe disease [21•]. Similar trends of increasing prevalence of CAMRSA are being observed from other parts of the world including Japan and India [23•, 24•]. Recently published reports regarding prevalence of CA-MRSA among isolates of S. aureus was $64 \%(28 / 44)$ in Japan and $46 \%(140 / 305)$ from India $[23 \bullet, 24 \bullet]$. Clindamycin resistance is another emerging problem among the cases of CA-MRSA [24•]. Furthermore, $p v l$ emerged as a significant predictor of need for a drainage procedure and higher number of surgical interventions, indicating that PVL (or an associated factor) likely contributes to severity of muscle disease caused by $S$. aureus.

In tropical countries, pyomyositis was said to occur in individuals who were malnourished and who had multiple parasitic infections. However, this association has not been confirmed in children with pyomyositis seen in India, the USA or Australia $[5 \cdot 15,25]$. Giasuddin AS et al. proposed that IgM antibody deficiency against $S$. aureus may be a cause of TP in Nigerians [26]. But extensive immunological evaluations in most of other studies have been normal, including quantitative immunoglobulins, enumeration of T-lyphocyte subpopulations, total haemolytic complement levels and leukocyte function as tested by reduction of nitroblue tetrazolium (NBT) [5•, 27]. Some other immune dysfunction states proposed to have association with TP are HIV infection, steroid use, diabetes mellitus, leukaemia and lymphoma. Tropical pyomyositis cases in immunocompromised patients are more commonly reported from temperate regions. But in the tropics, TP have been reported even in otherwise normal children too.

Other less common isolates in temperate climates could be Streptococcus pyogenes followed by Escherichia coli. Acute 
bacterial myositis, by group A streptococci, is far less common than classical (tropical) pyomyositis. It usually causes much diffused illness and more commonly seen in adults. Viral infection, most commonly influenza virus, occasionally leads to myositis, characterised by pain in the muscles, mainly the legs generally lasting 2 to 4 days. Typically, school-aged children are affected and serum creatine phosphokinase (CPK) shows marked elevation. Rarely, Coxsackie virus B can cause similar illness. Other uncommon causes of myositis could be Candida albicans (immunocompromised patients) and parasites like Trichinella spiralis.

\section{Clinical Presentation}

Although the classical presentation of TP is muscle abscess, the disease process passes through three stages. During the first stage (invasive stage), the infective muscle becomes inflamed and painful and there may be associated mild leukocytosis. Examination will reveal a woody muscle texture with no fluctuation, palpable abscess or surrounding erythema. The second stage (suppurative phase) involves formation of a muscle abscess where patients will usually have severe pain, worsening swelling and fever. Aspiration of the affected area would reveal purulent material. The majority of patients are diagnosed at this stage which lasts 1-3 weeks. The third stage involves systemic toxicity and septicaemia, multifocal abscess formation and shock [28, 29•].

Pyomyositis is often considered a disease of adolescents and young adults, although it occurs in all age groups including infants and young children. It is more commonly reported among boys, but girls engaged in sporting activities like athletics had equal chances of having it $[5 \cdot, 30]$. Unifocal presentation is common, but literature evidence suggests it can be multifocal in 15-43\% of cases [31]. Commonly involved sites are the muscles around the pelvis and lower limbs. The most common site of abscess formation is the thigh, followed by the calf, buttock, arm, scapula and chest wall (Table 1). Often, there is a history of having recent accidental injury (often involving the leg) that usually was not considered serious. After a few days, the child developed low-grade fever; symptoms persisted a few days to a few weeks until a mass appeared. Initial examination by physician, in many of the patients, was considered to have only a contusion or a haematoma (Table 1).

When pyomyositis involves the muscles of the pelvis, pain is usually transferred to the hip. Pelvic pyomyositis initially originates from the anterolateral and posterolateral walls of the pelvic cavity and surrounds the obturator foramen, ending in a tendinous band across the posterior capsule of the hip involving deep pelvic muscles like psoas, iliacus, piriformis and obturators [38]. One study from the USA showed high proportion of cases of pericapsular pyomyositis ( $32 \%$ ) among 53 children, initially referred as cases of septic arthritis/irritable hip joint [39•]. It is noteworthy that patients with pelvic pyomyositis may present with limited range of motion in a specific plane (the motion placing the infected muscle on stretch) vs global limited range of motion of the joint as is commonly seen in septic arthritis [35]. When the iliopsoas is affected, there will be a discrete presenting symptom of pain in the retroperitoneal muscle location. The limb affected will be held in thigh flexion and external rotation [14•]. Another usual clinical presentation could be acute abdominal pain in some of them, which often needs sonography for making the diagnosis. A review from Nigeria found muscle abscesses in the anterior abdominal wall to be common. Possibility of TP should also be considered in a child with hip pain and fever,

Table 1 Clinical spectrum and aetiology of pyomyositis cases

\begin{tabular}{|c|c|c|c|c|c|c|c|}
\hline Sl. no. & Study & No. of cases & $\begin{array}{l}\text { Males } \\
(\%)\end{array}$ & $\begin{array}{l}\text { Common muscle groups } \\
\text { involved }\end{array}$ & $\begin{array}{l}\text { Pus/blood culture } \\
\text { positivity (S. aureus) }\end{array}$ & $\begin{array}{l}\text { Proportion } \\
\text { of CA-MRSA }\end{array}$ & $\begin{array}{l}\mathrm{h} / \mathrm{o} \text { muscle } \\
\text { trauma }\end{array}$ \\
\hline 1. & Moriarty P. et al. [13••], Brisbane (2015) & 34 & $79 \%$ & Vertebra-pelvic & $65 \%$ & $4 / 22(18 \%)$ & - \\
\hline 2. & Verma S. et al. [5•], India (2013) & 40 & $68 \%$ & $\begin{array}{l}\text { Thigh }(58 \%), \operatorname{leg}(35 \%) \\
\text { psoas }(30 \%)\end{array}$ & $43 \%$ & - & $30 \%$ \\
\hline 3. & Borges AH. et al. [32], Brazil (2012) & 82 & - & Lower limb (64\%) & $90 \%$ & - & - \\
\hline 4. & Unnikrishnan PN. et al. [14•], UK (2010) & 13 & $54 \%$ & Obturator internus (62 \%) & $69 \%$ & - & - \\
\hline 5. & Chiu NC. et al. [33•], Taiwan (2009) & 24 & $54 \%$ & - & $58 \%$ & - & $33 \%$ \\
\hline 6. & $\begin{array}{l}\text { Pannaraj PS. et al. [21•], Texas, } \\
\text { USA (2006) }\end{array}$ & 45 & - & Thigh (40\%), pelvis (29\%) & $53 \%$ & $15 / 24(63 \%)$ & - \\
\hline 7. & Tanir G. et al. [34] Turkey (2006) & 06 & - & $\begin{array}{l}\text { Extremities }(83 \%) \text { trunk } \\
\quad(17 \%)\end{array}$ & $16 \%$ & - & - \\
\hline 8. & $\begin{array}{l}\text { Bertrand SL et al. [35] Georgia, } \\
\text { USA (2011) }\end{array}$ & 08 & & Obturator internus (63\%) & $88 \%$ & - & - \\
\hline 9. & Mitsionis G et al. [36] Greece (2009) & 06 & - & Hip and thigh & $50 \%$ & - & - \\
\hline 10. & $\begin{array}{l}\text { Brook I et al. [37] Washington, } \\
\text { USA (1996) }\end{array}$ & 06 & $67 \%$ & Gluteal (50 \%) & $\begin{array}{r}\text { Anaerobic } \\
\text { bacteria }\end{array}$ & - & - \\
\hline
\end{tabular}


after ruling out septic arthritis [40••]. Because of such atypical clinical presentations, it is important for physicians to be aware of this rare but potentially complicated condition.

Complications are more common when disease is diagnosed late. Common complications associated with TP include venous thrombosis, arthritis, osteomyelitis and pneumonia. In the late stage of disease, dissemination of $S$. aureus could lead to sepsis, which has potential to involve other organs and cause acute respiratory distress syndrome (ARDS), pericarditis, acute renal failure and even meningitis, but these are not seen often. Common complications reported, in a review from Northern India, were arthritis ( $25 \%)$, pneumonia $(18 \%)$ and osteomyelitis (5\%) [5•]. Arthritis in most of them was reactive (sterile), which recovered spontaneously, while 2 out of 40 children had purulent arthritis which needed drainage [5•]. Recent report from Texas, USA, showed associated osteomyelitis (41\%) and concomitant septic arthritis (7\%) to be the commonest complications associated with TP [21•]. S. aureus strains that carried pvl were associated with increased levels of inflammatory markers, longer hospitalizations and more complications [21•].

\section{Diagnosis}

Diagnosis of pyomyositis should be considered in any child presenting with fever and acute muscle pain, especially in season of high temperature and high humidity and when history of recent trauma exists. When a child has visible masses at commonly involved sites, like the lower limb muscles, the diagnosis of pyomyositis can be made by needle aspiration of a mass. A definitive diagnosis usually depends on one or more radiologic procedures, especially when there is fever and myalgia, but no palpable mass. Plain films may show a soft tissue swelling or even a widened fascial plane suggestive of a mass lesion, seldom useful in the diagnosis but appropriate to exclude any suspected bony pathology. Ultrasounography is used commonly in emergency for diagnosis of muscle masses and to guide percutaneous aspiration for culture and drainage. Ultrasounography may be used as the first line since it is noninvasive, easily obtainable and inexpensive [14•].

For definitive diagnosis, MRI is preferred to $\mathrm{CT}$ as it is able to delineating the extent of a muscle abscess. In many cases, the abscess is much larger than suspected by clinical examination. MRI typically shows hyperintense signal in T2 weighted images, hyperintense rim on enhanced T1 weighted images. For the reasons described, MRI has become the preferred procedure for establishing the diagnosis of pyomyositis [41-43]. Blood investigations may show anaemia, leukocytosis (shift to left) raised erythrocyte sedimentation rate and acute-phase reactants [5•]. Muscle enzymes are always normal; this finding serves to differentiate TP from polymyositis. Bone scintigraphy lacks sensitivity to identify pyomyositis but can show any associated osteomyelitis. Pus culture positivity rates are often high, which gives the aetiological agent. Blood culture positivity rates are often low comparatively $[5 \bullet, 44]$.

\section{Treatment}

Once diagnosed, treatment of pyomyositis consists of appropriate antibiotics and possible abscess drainage depending on the size and pressure symptoms. In early stages, TP may be treated conservatively with antibiotics alone or antibiotics and percutaneous aspiration. However, a surgical decompression may subsequently be required in $50 \%$ of them [45 $]$. An important role of systemic antibiotics is to prevent the formation of further abscesses, especially in children with proven bacteraemia. Empirical antimicrobial therapy for pyomyositis will depend upon local epidemiologic and susceptibility patterns. Because $S$. aureus is the most likely agent, a semisynthetic penicillinase-resistant penicillin should be the preferred antibiotic in communities with relatively little MRSA. Usually, nafcillin or oxacillin 150 to $200 \mathrm{mg} / \mathrm{kg}$ can be administered intravenously every $6 \mathrm{~h}$. Cloxacillin at similar dosages is equally effective, commonly used in India [14•].

In many countries including India, community-acquired MRSA (CA-MRSA) is now a common agent, to the tune of around $50 \%$ (Table 1 ). In settings where CA-MRSA is prevalent in significant proportions, one should strongly consider coverage for CA-MRSA in the empirical treatment regimen [46•]. Although literature does not support the use of more than one sensitive drug for the treatment of $S$. aureus, in the present scenario, it would be wise to use combination of antistaphylococcal antibiotics, initially. The number of sensitive drug could be minimised once culture and sensitivity reports are available. In India, the choices for empirical therapy could be clindamycin alone, cloxacillin along with clindamycin or a combination of cloxacillin, clindamycin and amikacin. Combination therapy should be stressed more especially in a critically ill child having fulminant sepsis, as a complication of TP by $S$. aureus. Clindamycin may have a role in cases of acute bacterial myositis of malignant type because of the Eagle effect, which showed superiority of clindamycin over penicillin in the treatment of streptococcal myositis [47]. Bickles et al. recommended cloxacillin with the addition of aminoglycoside if patients are immunocompromised or in a state of sepsis [47]. Zimbelman et al. recommend clindamycin with or without penicillin as superior to B lactamase antibiotic alone [48•]. Clindamycin $40 \mathrm{mg} / \mathrm{kg} /$ day divided every $8 \mathrm{~h}$ could be a reasonable option also for penicillin hypersensitivity. Vancomycin, teicoplanin and linezolid are the options for clindamycin-resistant CA-MRSA. Dose of vancomycin in children should be $60 \mathrm{mg} / \mathrm{kg} /$ day divided every $6 \mathrm{~h}$, and in adolescents, dose is usually reduced to $45 \mathrm{mg} / \mathrm{kg} /$ day, divided every $8 \mathrm{~h}$. Where available, a glycopeptide antibiotic closely 
related to vancomycin called teicoplanin can be substituted for vancomycin; the teicoplanin loading dosage is $10 \mathrm{mg} / \mathrm{kg} \mathrm{IV}$ every $12 \mathrm{~h}$ for three doses, followed by $10 \mathrm{mg} / \mathrm{kg}$ once daily [49]. Both nephrotoxicity and red-man syndrome are less common after teicoplanin than vancomycin.

Parenteral therapy is continued until clinical improvement is evident, usually within a few days after surgical drainage. Thereafter, the antibiotics can be given orally for an additional 2 to 3 weeks. For methicillin-sensitive isolates, oral cloxacillin, cephalosporin (cephalexin $100 \mathrm{mg} / \mathrm{kg} / \mathrm{day}$ ) or clindamycin 30 to $40 \mathrm{mg} / \mathrm{kg} /$ day is acceptable [50]. For CA-MRSA isolates, clindamycin can be continued as an oral antibiotic. For MRSA isolates resistant to clindamycin, intravenous treatment can be followed by administration or oral linezolid $30 \mathrm{mg} / \mathrm{kg} /$ day divided every $8 \mathrm{~h}$ for children younger than 12 years; for children 12 years and older, $20 \mathrm{mg} / \mathrm{kg}$ /day divided every $12 \mathrm{~h}$ is given. Therapy of MRSA infection may require a longer duration, from 3 to 4 weeks. Before discontinuing antibiotics, the patient should have documented normal levels of C-reactive protein and erythrocyte sedimentation rate. Since there are reports of anaerobic bacteria causing pyomyositis in children; appropriate anaerobic cover should be added in cases where such organisms are suspected or isolated [37].

Treatment options for VRSA (MIC $\geq 16 \mu \mathrm{g} / \mathrm{ml}$ ) and VISA (MIC $4-8 \mu \mathrm{g} / \mathrm{ml}$ ) include IV infusion of vancomycin and IV daptomycin, which might be tried even in children. In such cases, loading dose of vancomycin $25-30 \mathrm{mg} / \mathrm{kg}$ may be considered; continuous infusions have been found more effective. Daptomycin (bactericidal against MRSA) was used in 15 children at Dallas, USA, in sever MRSA infection, in a dose of 4-6 $\mathrm{mg} / \mathrm{kg} /$ day for a duration of 6-36 days (median 10 days) with good clinical response. It was well tolerated and presently, an RCT is ongoing for this in children. In these cases, extended drug sensitivity should be asked; if sensitive, addition of drugs like fluoroquinolones, cotrimoxazole, doxycycline $(>8$ years age) or rifampicin might be useful.

Complications are more with this potentially lifethreatening illness if diagnosed late. Continuation or recurrence of fever after surgical drainage while the patient is receiving appropriate antimicrobials suggests the presence of other foci needing further drainage, development of drug resistance or, less commonly, drug fever. Early diagnosis is associated with good prognosis in this curable infective disease entity. Although there are no recommendations to prevent pyomyositis, it is believed that nasal carriage should be eliminated in patients with a history of pyomyositis or bacteraemic staphylococcal infection. It should be treated with topical mupirocin nasal formulation. Alternatives include rifampicin or cloxacillin for 10 days [32, 34, 36, 51].

\section{Conclusion}

Pyomyositis is primarily a disease of the tropics, showing increasing incidence in temperate regions too. S. aureus remains the primary aetiological agents, although CA-MRSA and clindamycin resistance is on rise, worldwide. Choice of empirical antibiotics remains debatable and should be guided by prevalent resistance patterns. Timely diagnosis, right choice of antibiotics and appropriate interventions remain the key to success in management of TP. A high level of awareness and suspicion of this condition is warranted from all paediatric clinicians.

\section{Compliance with Ethical Standards}

Conflict of Interest Dr. Hede declares no competing interests.

Human and Animal Rights and Informed Consent This article does not contain any studies with human or animal subjects performed by the author.

\section{References}

Papers of particular interest, published recently, have been highlighted as

- Of importance

•- Of major importance

1. Scriba J, Beitrang Z. Aetiologie der myositis acuta. Deutsche Zeit Chir. 1885;22:497-502.

2. Miyake H. Beitragezurkenntnis der sogenannten myositis infectiosa. Mitt Grenageb Med Chir. 1904;13:155-98.

3. Ashken MH, Cotton RE. Tropical skeletal muscle abscesses (pyomyositis tropicans). Br J Surg. 1963;50:846-52.

4.• Groose C. Bacterial myositis and pyomyositis. In: Cherry JD, Harrison GJ, Kaplan SL, Steinbach WJ, Hotez PJ, editors. Feigin and Cherry's textbook of pediatric infectious diseases. 7th ed. Philadelphia: Elesvier Saunders; 2014. This article shows us the epidemiology of pyomyositis in world, one of the most useful reviews on pyomyositis published in textbook of pediatric infectious diseases.

5. Verma S, Singhi SC, Marwaha RK, et al. Tropical pyomyositis in children: 10 years experience of a tertiary care hospital in northern India. J Trop Pediatr. 2013;59(3):243-5. One of the biggest case series from India about tropical pyomyositis in pediatric age group.

6. Gambhir IS, Singh DS, Gupta SS, Gupta PR, Kumar M. Tropical pyomyositis in India: a clinico-histopathological study. J Trop Med Hyg. 1992;95(1):42-6.

7. Malhotra P, Singh S, Sud A, et al. Tropical pyomyositis-experience of a tertiary care hospital in North West India. J Assoc Physicians India. 2000;48:1057-60.

8. Chauhan S, Kumar R, Singh KK, Chauhan SS. Tropical pyomyositis: a diagnostic dilemma. J Ind Acad Clin Med. 2004;5:52-4.

9. Chauhan S, Jain S, Varma S, Chauhan SS. Tropical pyomyositis (myositis tropicans): current prospective. Postgrad Med J. 2004;80: 267-70.

10. Christin L, Sarosi GA. Pyomyositis in North America: case reports and review. Clin Infect Dis. 1992;15:668-77. 
11. Gibson RK, Rosenthal SJ, Lukert BP. Pyomyositis. Increasing recognition in temperate climates. Am J Med. 1992;77:768-72.

12. Hall RI, Callaghan JJ, Moloney E, et al. Pyomyositis in a temperate climate. Presentation, diagnosis, and treatment. J Bone Joint Surg Am. 1990;72:1240-4.

13.• Moriarty, Leung C, Walsh M, Nourse C. Increasing pyomyositis presenting among children in Queensland, Australia. Pediatr Infect Dis J. 2015;34(1):1-4. Recent report of increasing incidence of pyomyositis from Australia.

14. Unnikrishnan PN, Perry DC, George H, Bassi R, Bruce CE. Tropical primary pyomyositis in children of the UK: an emerging medical challenge. Int Orthop. 2010;34:109-13. Recent report of pyomyositis from UK, showing prevalence in United Kingdom.

15. Gubbay AJ, Isaacs D. Pyomyositis in children. Pediatr Infect Dis J. 2000;19(10):1009-12.

16. Spiegel DA, Meyer JS, Dormans JP, Flynn JM, Drummond DS. Pyomyositis in children and adolescents: report of 12 cases and review of the literature. J Pediatr Orthop. 1999;19(2):143-50.

17. Horn CV, Master S. Pyomyositis tropicans in Uganda. E Afr Med J. 1968;45(7):463-71.

18. Smith MI, Vickers AB. Natural history of 338 treated and untreated patients with staphylococcal septicaemia (1936-1955). Lancet. 1960;1(7138):1318-22.

19. Jayoussi R, Bialik V, Eyal A, Shehadeh N, Etzioni A. Pyomyositis caused by vigorous exercise in a boy. Acta Paediatr. 1995;84(2): 226-7.

20. Singh SB, Singh VP, Gupta S, Gupta RM, Sunder S. Tropical myositis: a clinical, immunological and histopathological study. J Assoc Physicians India. 1989;37(9):561-3.

21. Pannaraj PS, Hulten KG, Gonzalez BE, Mason Jr EO, Kaplan SL. Infective pyomyositis and myositis in children in the era of community-acquired, methicillin-resistant Staphylococcus aureus infection. Clin Infect Dis. 2006;43:953-60. This article emphasizes the changing epidemiology of causative organisms of tropical pyomyositis.

22. Mitchell PD, Hunt DM, Lyall H, Nolam M, et al. Panton-Valentine leukocidin-secreting Staphylococcus aureus causing sever musculoskeletal sepsis in children. A new threat. J Bone Joint Surg (Br). 2007;89:1239-42. This articles shows us the association of PVL genes with pyomyositis.

23. Yamaguchi T, Nakamura I, Chiba K, Matsumoto T. Epidemiological and microbiological analysis of communityassociated methicillin-resistant Staphylococcus aureus strains isolated from a Japanese hospital. Jpn J Infect Dis. 2012;65(2):175-8. This article is one of the biggest reviews on pyomyositis in Japanese population.

24. Lall M, Sahni AK. Prevalence of inducible clindamycin resistance in Staphylococcus aureus isolates from clinical samples. Med J Armed Forces India. 2014;70(1):43-7. This article state the increasing prevalence of clindamycin resistance in staphylococcus aureus.

25. Diamandakis V, Grose C. Bad consequences of bicycle accidents. Pyomyositis. Pediatr Infect Dis J. 1994;13(5):422-5.

26. Giasuddin AS, Idoko JA, Lawande RV. Tropical pyomyositis: is it an immunodeficiency disease? Am J Trop Med Hyg. 1986;35(6): $1231-4$.

27. Ladipo GO, Fakunle YF. Tropical pyomyositis in the Nigerian Savanna. Trop Geogr Med. 1977;29(3):223-8.

28. Crum-Cianflone NF. Infection and musculoskeletal conditions: infectious myositis. Best Pract Res Clin Rheumatol. 2006;20:1083-97.

29. Hadjipavlou M, Butt DA, McAllister J. Primary pyomyositis: an unusual presentation in an older patient with no recognised risk factors. BMJ Case Rep. 2012. doi:10.1136/bcr.12.2011.5342. This article emphasizes that pyomyositis is possible even in person without any risk factors.

30. Meehan J, Grose C, Soper RT, Kimura K. Pyomyositis in an adolescent female athlete. J Pediatr Surg. 1995;30(1):127-8.
31. Chiedozi LC. Pyomyositis. Review of 205 cases in 112 patients. Am J Surg. 1979;137:255-9.

32. Borges AH, Faragher B, Lalloo DG. Pyomyositis in the upper Negro river basin, Brazilian Amazonia. Trans R Soc Trop Med Hyg. 2012;106(9):532-7.

33. Chiu NC, Hsieh MC, Chi H, Huang FY. Clinical characteristics of pyomyositis in children: 20-year experience in a medical centre in Taiwan. J Microbiol Immunol Infect. 2009;42(6):494-9. Recent articles stating pyomyositis in hip area in children.

34. Tanir G, Tonbul A, Tuygun N, Aydemir C, Ertan U. Soft tissue infections in children: a retrospective analysis of 242 hospitalized patients. Jpn J Infect Dis. 2006;59(4):258-60.

35. Bertrand SL, Lincoln ED, Prohaska MG. Primary pyomyositis of the pelvis in children: a retrospective review of 8 cases. Orthopedics. 2011;34(12):832-40.

36. Mitsionis G, Manoudis GN, Lykissas MG, Sionti I, Motsis E, Georgoulis AD, et al. Pyomyositis in children: early diagnosis and treatment. J Pediatr Surg. 2009;44(11):2173-8.

37. Brook I. Pyomyositis in children, caused by anaerobic bacteria. J Pediatr Surg. 1996;31(3):394-8.

38. Garcia-Mata S, Hidalgo-Ovejero A, Esparza-Estaun J. Primary obturator-muscle pyomyositis in immunocompetent children. J Child Orthop. 2012;6:205-15.

39. Mignemi ME, Menge TJ, Cole HA, et al. Epidemiology, diagnosis, and treatment of pericapsular pyomyositis of the hip in children. J Pediatr Orthop. 2014;34(3):316-25. Articles emphasizing role of imaging in diagnosing pyomyositis.

40.• Leuthauser A, Paul A. Hip pain and fever: when it's not a septic joint, what's next? Pediatr Emerg Care. 2015;31(1):42-3. This report emphasizing cases of spinal pyomyositis and role of imaging.

41. Solar R, Rodriguez E, Aguiler C, Fernandez R. Magnetic resonance imaging of pyomyositis in 43 cases. Eur J Radiol. 2000;35(1):59 64.

42. Trusen A, Beissert M, Schultz G, Darge K. Ultrasound and MRI features of pyomyositis in children. Eur Radiol. 2003;13(5):1050-5.

43. Moran GG, Duran CG, Albinana J. Imaging on pelvic pyomyositis in children related to pathogenesis. J Child Orthop. 2009;3:479-84.

44. Adamski GB, Garin EH, Ballinger WE, et al. Generalized nonsuppurative myositis with staphylococcal septicemia. J Pediatr. 1980;96:694-7.

45. Boulyana M, Kilani MS. Nontropical pyomyositis complicated with spinal epidural abscess in a previously healthy child. Surg Neurol Int. 2014;5:119-21. Article emphasizing role of clindamycin in treatment of pyomyositis.

46. Vander Have KL, Karmazyn B, Verma M, et al. Communityassociated methicillin-resistant Staphylococcus aureus in acute musculoskeletal infection in children: a game changer. J Pediatr Orthop. 2009;29(8):927-31. Article explaining eagle effect in management of staphylococcal infections.

47. Brickels J, Ben-Sira L, Kessler A, Wientroub S. Primary pyomyositis. J Bone Joint Surg Am. 2002;84:2277-86.

48. Zimbelman J, Palmer A, Todd J. Improved outcome of clindamycin compared with beta-lactam antibiotic treatment for invasive Streptococcus pyogenes infection. Pediatr Infect Dis J. 1999;18: 1096-100. This article showing anaerobes to be causative organism for pyomyositis.

49. Svetitsky S, Leibovic L, Paul M. Comparative efficacy and safety of vancomycin versus teicoplanin. Antimicrob Agents Chemother. 2009:53(10):4069-79.

50. Stevens DL, Gibbons AE, Bergstrom R, Winn V. The Eagle effect revisited: efficacy of clindamycin, erythromycin, and penicillin in the treatment of streptococcal myositis. J Infect Dis. 1988;158:23-8.

51. Hudson IR. The efficacy of intranasal mupirocin in the prevention of staphylococcal infections: a review of recent experience. J Hosp Infect. 1994;27:81-98. 